\title{
Training in robotic surgery, replicating the airline industry. How far have we come?
}

\author{
Justin William Collins ${ }^{1,2,4} \mathbb{\oplus} \cdot$ Pawel Wisz $z^{2,3}$
}

Received: 24 May 2019 / Accepted: 20 August 2019 / Published online: 17 October 2019

(c) The Author(s) 2019

\begin{abstract}
Introduction As the role of robot-assisted surgery continues to expand, development of standardised and validated training programmes is becoming increasingly important. We aim to compare current robotic training curricula with training in aviation, to evaluate current similarities and to provide insight into how healthcare can further learn from replicating initiatives in aviation training.

Methods A systematic literature review of the current evidence was conducted online and relevant publications and information were identified. Evaluation and comparison between training in robotic surgery and the aviation industry was performed. Results There are significant similarities between modern robotic training curricula and pilot training. Both undergo basic training before proceeding to advanced training. Aviation training methods include classroom instruction, e-learning and practical training, in both the aircraft and flight simulation training devices. Both surgeon and pilot training include technical and procedural instruction as well as training in non-technical skills such as crisis management, decision making, leadership and communication. However, there is more regulation in aviation, with international standards for training curricula, simulation devices and instructors/trainers that are legally binding. Continuous learning with re-qualification with benchmarked high stakes tests are also mandatory throughout a pilot's and instructor's career.

Conclusion Robotic surgeons and pilots roles have many fundamental similarities. Both work with expensive and complex technology requiring high levels of skills, within working environments with high physiological and psychological stress levels. Whilst many initiatives in aviation training have already been replicated in surgical training there remain considerable differences in regulation. Adopting established and proven aviation methods of assessment and regulation could help robotic surgical training become more efficient, more effective and ultimately safer.
\end{abstract}

Keywords Robotic-assisted surgery $\cdot$ Training $\cdot$ Non-technical skills $\cdot$ Surgical education $\cdot$ Patient safety $\cdot$ Proficiencybased progression

\section{Introduction}

The airline pilot training model has often been used as an analogy for improved surgical training [1]. For pilots, training methods include classroom instruction, e-learning and

Justin William Collins

justin.collins@ki.se

1 Department of Molecular Medicine and Surgery, Karolinska Institutet, Stockholm, Sweden

2 Orsi Academy, Melle, Belgium

3 OLV Hospital, Aalst, Belgium

4 Department of Uro-oncology, UCLH (University College London Hospital), London, UK practical training, in both the aircraft and with flight simulation training. Pilot training focuses not only on technical and procedural instruction, but also training in non-technical skills, including crisis (emergency) management, decision making, leadership and communication, that benefits the team performance. Airline pilot training has many similarities with robotic surgery training with both having basic training before proceeding to advanced training. In robotic surgery advanced training relates to specialty-specific procedural training and for pilots, it relates to a specific aircraft type. In the near future, when multiple robotic surgical platforms become available, the need to 'retrain' in platform (device)-specific training modules will also become a further area of commonality. 
The structure to training in aviation and robotic surgery has many similarities and both parties control complex technology with their hands, that if managed inappropriately could result in fatal consequences. However, the safety outcome records compared between the healthcare and aviation industries are very different. In the book 'Why Hospitals should fly', the author J Nance reported mortality comparisons between the aviation and healthcare industries over a 5-year period between 2001 and 2006, with zero deaths on commercial US flights compared to an estimated 250-500,000 deaths from medical errors in the US healthcare system at the same time [2].

In the United States between 2000 and 2013, 10,624 adverse events related to robotic procedures were reported [3]. In 2013, a group of experts expressed concern that robotic surgery training is random and insufficient to ensure patient safety [4]. Two years later the Emergency Care Research Institute (ECRI) institute on health technology hazards published an independent review in which a lack of robotic surgical training as 1 of the top 10 risks to patients was identified [5].

An important aspect that differs between aviation and surgical training is the increased standardisation with internationally agreed training standards in the airline industry. Evaluation and regulation comprise of benchmarked high stakes tests related to proficiency-based training that results in quality assurance [6]. Aviation training curricula, simulation training devices and instructors/trainers are standardised throughout the world and these standards are legally binding. Re-qualification and recurrent training to defined benchmarked metrics of performance are mandatory at all stages of a pilot's and instructor's career.

A second aspect that differs between the two industries is the focus on 'systems thinking' compared to focusing on individuals. System thinking addresses the 'system' or organisation as a whole and is key to the success of highreliability organisations (HRO), such as aircraft carriers. Similarly, to the aviation industry, there are multiple factors involved in robotic surgical training that increase risks to patient safety, these include patient factors, provider factors, task factors, technology and tool factors, team factors, environmental factors and organisational factors. Surgery has multiple safety variables to manage simultaneously within an organisation and yet surgical errors have often been managed with a focus on the individual surgeon, with an approach of 'name, blame, shame and retrain' [7].

In a high-reliability organisation (HRO), safety and quality (SQ) is an organisational priority, and all workforce members are engaged, continuously learning and improving their work. To build organisational capacity for SQ work, some healthcare organisations have developed capacitybuilding framework with a specific focus on patient safety and defined quality outcome measures, as part of an organisational strategy towards HRO [8].

A framework considering organisation-wide competencies for SQ includes all staff and faculty and is integrated into a broader organisation-wide operating management system for continuously improving quality. Achieving safe, high-quality care within this framework, is directly related to healthcare workforce training. In an article by Reason, he evaluated the cause of errors in healthcare utilising a system's thinking approach. Describing the 'Swiss cheese model', Reason identified that healthcare errors are a combination of active failures related to individuals and also latent failures related to the overall healthcare system architecture [9]. Reason's solution to improving patient safety was 'systems thinking' approach describing four increasing 'barriers' of defence, namely: (1) policy writing and training, (2) standardisation and simplification, (3) automation, and (4) improvement to devices and architecture.

In this paper, we assess and compare established practices in the aviation industry, that follow Reason's principles, compared with current approaches to training in robotic surgery. Identifying what are the current similarities and also opportunities to evolve robotic training based on the evidence from established and proven aviation industry training practices. The review includes an assessment of how much of a 'system's thinking' approach has been integrated into robotic surgery training and where the potential areas of improvement are for the future.

\section{Materials and methods}

A systematic narrative review was performed with a comprehensive computerised search completed using PubMed and Medline databases. We systematically searched using subject headings including 'robot-assisted surgery training', 'robotic surgery training', 'curriculum development' and 'proficiency-based training', 'pilot simulation training', 'aviation training', and 'pilot curriculum'.

Articles of interest included reports comparing aviation training with healthcare, prospective studies on the impact of robotic simulation training, robotic training curriculum development with validation and systematic reviews on robotic training published between July 2000, when the first robotic systems received FDA approval in the US [10] - and May 2019. Other significant studies cited in the reference list of selected papers were evaluated, as well as studies of interest published before the systematic search.

Two reviewers independently selected papers for detailed review evaluating the abstract and, if necessary, the full-text manuscript. Potential discrepancies were resolved by open discussion. 


\section{Findings}

The electronic search yielded a total of 253 potential articles. Following abstract review, 104 were critically reviewed for evidence synthesis. Overall, the quality of available studies was found to be low. Available evidence consists largely of expert opinion, consensus statements and small qualitative studies. There were no publications identified that focused on international standards for regulation within robotic-assisted surgery training. The review identified that there are many areas in robotic surgery and aviation training that share common approaches. Both have baseline evaluations, e-learning and simulation-based training and there is an increasing focus on non-technical skills training in robotic surgery [11]. Both industries also use checklists and automation to assess performance in real time. There was found to be significant time delays between the introduction of training initiatives in surgical training compared to the aviation industry. In Table 1, we have summarised the areas of commonality, categorising them under the headings of Reason's 'barriers' to patient safety.

\section{Checklists}

Aviation checklists were introduced in 1920. Aviation checklists are divided into subsections with defined tasks that focus on critical segments such as takeoff, approach, and landing. Similar to the approach of the World Health Organisation (WHO), checklist which focuses on defined significant time points to complete the checklist, i.e., before induction, before skin incision and before the patient leaves the OR. Checklists have been introduced relatively recently into healthcare with the WHO 'Surgical safety' checklist, launched on June 25 2008 [12]. The implementation of the WHO checklist into the operating theatres was shown to result in a significant decrease in peri-operative mortality from 1.5 to $0.7 \%$ and inpatient complications from 11.0 to $7.0 \%$ [17].

\section{Training curriculum development}

The first validated robotic training curriculum was published in 2015 [13]. It consists of various stages that are progressively completed with the trainee required to successfully pass each stage before progressing to the next, including e-learning, baseline evaluation, OR observation and first assistant training. Followed by a specialist attachment, such as a 'fellowship' at a high volume centre of expertise, with modular training, progressing to full procedure training and final assessment [13]. There is increasing awareness of the importance and benefits of standardised robotic surgery training and multiple specialist curricula have recently been developed by international panels of experts [18]. We are also seeing publications on guidance for procedural training [19].

E-learning is well established in the aviation industry. There is increased awareness of the benefits of e-learning in healthcare, in both increasing access to learning and driving standardisation in curricula content. There is also growing evidence on the effectiveness of e-learning on clinician performance and patient outcomes [20].

Non-technical skills training, error identification and crisis management have been key elements of aviation training for decades and there is evidence of increasing awareness and adoption of these important areas of robotic training $[11,21]$. However, it is recognised that often robotic training curriculums currently prioritise technical skills training and lack training in the area of non-technical skills [22].

\section{Simulation training}

Simulation can be defined as "a technique to replace or amplify real experience with or without guidance, often immersive in nature, that evokes or replicates aspects of the real world in a fully interactive manner" [15]. The first flight simulator was built in 1929 by Edwin Link. Driven by the cost of aircrafts and the financial and moral obligations to preserve human lives of both employees and customers, the airline industry has invested heavily in developing and standardising VR simulation training. Modern aviation simulators follow internationally agreed standards, are of high fidelity and can be utilised to train pilots in both standard and extreme conditions.

Table 1 Summary of initiative from aviation that have been replicated in surgical training

\begin{tabular}{llll}
\hline Description (level of barrier) & Airline industry & Healthcare system & $\begin{array}{l}\text { Approximate delay } \\
\text { to initiation (years) }\end{array}$ \\
\hline Policy writing training (level 1) & The first checklist 1920 & The WHO checklist 2008 [12] & 88 \\
Standardisation and simplification (level 2) & The first pilot license 1927 & The first validated curriculum 2015 [13] & 88 \\
Simulation (level 2) (VR simulation level 3) & The first flight simulator 1920 & The first VR simulation system 1993 [5] & 73 \\
Automation (level 3) & The first Black box 1953 & VR simulation 1993 [5] & Automated performance metrics [14] \\
& & OR Black box 2018 [15] & 65 \\
Better devices and architecture (level 4) & The first control tower 1920 & The first telementoring service 2001 [16] & 81 \\
\hline
\end{tabular}


The history of surgical simulation can be traced back more than 2500 years when leaf and clay models were used to conceptualise nasal reconstruction with a forehead flap [23]. The current use of robotic surgery simulators allows trainees to develop the basic surgical skills outside of the operating theatre and enables better patient safety and standards of care [16]. VR simulation modules are now routinely used in the baseline evaluation of trainees [13]. We are also seeing the first examples of full procedure training in VR simulation. The efficacy of VR simulation is currently limited by the lack of comparative studies, standardisation of validation and high costs of development and purchase price [24]. Due to these current issues with VR simulation other forms of simulation are utilised in robotic training curricula. Table 2 summarises the current advantages and disadvantages of various simulation models.

\section{Air traffic control towers and telementoring services}

Another area of support and enhanced feedback for pilots is the air traffic control tower. Telementorship programs have aimed to replicate this increased accessibility to expertise [28]. In 2001, the world's first national telesurgery initiative was launched in Canada with the goal of disseminating expertise from large tertiary hospitals to remote and rural medical centres [28-30]. The Centre for Minimal Access Surgery group led by Dr. Mehran Anvari, located at McMaster University and St. Joseph's Hospital in Ontario, Canada, trained surgeons through telementoring, utilising a dedicated Virtual Private Network
(VPN) [29, 30]. Studies confirmed benefits to training and patient outcomes [31].

\section{The black box and automated performance metrics}

The first 'black box' to record inflight data was first installed in 1953. This approach to automatically collect data from multiple recording devices to aid analysis and learning has recently been employed with the instillation of an operating room 'Black Box'. The study identified 'frequent intraoperative errors and events, variations in surgeon's technical skills and a high amount of environmental distractions', using the OR Black Box [32].

In a separate study, using a novel recorder of data taken directly from the da Vinci ${ }^{\circledR}$ Surgical System ("dVLogger"), Hung et al. published evidence that objective surgeon performance metrics can be captured. Showing construct and concurrent validation of automated performance metrics (APMs) during robotic surgery [33].This study further highlights the potential of collecting automated data during robotic surgery.

\section{Discussion}

Historically, many initiatives employed in aviation to improve training, data performance collection and enhanced feedback of performance have been replicated in surgical training. Often these occur many decades after evidence for these approaches has shown clear benefits to safety and quality in aviation (Table 1).

Table 2 Summary of simulation models currently used in surgical training

\begin{tabular}{|c|c|c|}
\hline Model & Strengths & Weaknesses \\
\hline Task deconstruction models & $\begin{array}{l}\text { Address metrics and are cost effective, e.g., Chicken gizzard model } \\
\text { for vesico-urethral anastomosis [25] }\end{array}$ & $\begin{array}{l}\text { Limited development to comprehensively } \\
\text { address metrics, benchmarks and error } \\
\text { management }\end{array}$ \\
\hline Porcine model & Flexible training model for tissue handling & $\begin{array}{l}\text { Expensive } \\
\text { Not human anatomy } \\
\text { No human pathology } \\
\text { Limited accessibility }\end{array}$ \\
\hline Canine cadaver model & Flexible training model for tissue handling & $\begin{array}{l}\text { Not human anatomy } \\
\text { No human pathology } \\
\text { Limited accessibility }\end{array}$ \\
\hline Human cadaver model & Flexible training model & $\begin{array}{l}\text { Expensive } \\
\text { Lacks human pathology and does not bleed } \\
\text { Limited accessibility }\end{array}$ \\
\hline 3D printed models & $\begin{array}{l}\text { Flexible training model } \\
\text { Can incorporates pathology and vascularisation } \\
\text { Increasingly realistic tissue handling } \\
\text { Can incorporate metrics and benchmarks [26] }\end{array}$ & $\begin{array}{l}\text { Currently, high development costs (low- } \\
\text { ered if printed casts rather than printed } \\
\text { models) } \\
\text { Models that address specific defined met- } \\
\text { rics need to be developed }\end{array}$ \\
\hline VR simulation & Advanced procedural training models available (e.g., RARP, RAH) & Current scope/range/image quality limited \\
\hline AR simulation & Potential to develop [27] & Limited development \\
\hline
\end{tabular}


The aviation industry has successfully navigated the hurdles to integrated training and data collection with international agreement on standards and high-stake tests that result in quality assurance. Standardised robotic surgery curricula have been shown to be beneficial in both delivering education and identifying trainees who have not reached the pre-requisite skillets [6]. However, surgical curricula do not currently have the same levels of regulation or international standards compared to aviation. To achieve standards that are adopted internationally requires consensus on curricula content and structure. As the role of robotic-assisted (computer-assisted) surgery continues to expand, there are increasing opportunities to replicate the aviation industry technology-driven approaches. There is also increased need for this. As new robotic platforms enter the clinical scene, the development of standardised and validated training programs is increasingly important. Curricula development is a crucial step in the global standardisation of training, accreditation and certification of surgeons for robotic surgical procedures.

Further work is needed in the development, validation and implementation of these programmes to reduce variability within training [24]. The integration of validated VR simulation devices will likely aid the development of international standards [34]. Another highly successful approach to drive training standards, employed in aviation, is the use of qualified and regulated instructors. Train the trainer initiatives in robotic surgery have adopted this principle and can similarly drive standardisation with a 'top down' approach [35]. There is also increasing focus in train-the-trainer courses to recognise objective outcome metrics that will enable proficiencybased progression training [3].

From Reason's defined four barriers to increasingly improving patient safety, we can see good evidence for the implementation of robotic training and policies to improve quality and safety such as the WHO checklist (1st level). There is increasing adoption of standardised robotic training curricula (2nd level). The third level comes from automation and in training we see evidence for this in VR simulation training modules with automated scoring systems and recently with automated performance metrics [33] and initiative such as the OR Black Box [32]. An advantage of VR simulator training over other simulation models is their ability to create objective performance data. Real-time feedback is provided, and results can be compared with validated data [24]. VR simulation training can, therefore, be practiced independently after suitable instruction. There are a number of robotic surgery training curricula developed in recent years which successfully include simulation training [13]. Both basic training and advanced procedural VR simulation training should be incorporated into robotic training curricula to shorten the learning curve without compromising patient safety. To enable this, we need cost-effective, high-fidelity, validated simulators to become incorporated into standardised, validated robot-assisted surgery training curriculum [34]. The development of more sophisticated training simulators that can accurately assess surgeon's performance with objective metrics as well as automated collection of performance metrics (Hung et al.) will ultimately evolve current training curricula and enable international credentialing standards, as they have in aviation.

Reason's 4th level barrier is improvement to devices and system architecture. There are similarities between control towers in aviation and telementoring services in surgery. Connecting training centres to educational hubs and telementoring services will result in robotic network development [36]. The potential and effectiveness of telementoring as an educational tool has been demonstrated, but the potential benefits of integration of telementoring and APMs into robotic training curricula to improve training system architecture have not yet been realised. This is partly due to issues related to setup costs and potential legal, data collection and ethical issues. Robotic network development would further enable automated data collection and comparison of outcomes. In the future, APMs could also result in device improvement with real-time alerts given by the surgical devices to help prevent surgical errors and events in the OR.

\section{Conclusions}

Robotic surgeons and pilot roles have many fundamental similarities. Both work with expensive and complex technology requiring high levels of skills, within working environments that have high physiological and psychological stress levels. Historically many initiatives in aviation training have been replicated in surgical training, but there remain considerable differences in regulation between healthcare and aviation. Better understanding of the impacts of training and the ability to accurately and reliably measure surgical performance with objective metrics, will enable systems thinking in robotic surgery training. Adopting established and proven aviation methods of assessment and regulation could help robotic surgical training become more efficient, more effective and ultimately safer.

Acknowledgements Open access funding provided by Karolinska Institute.

Author contributions JWC: project development, data collection, and manuscript writing/editing. PW: data collection and manuscript writing

\section{Compliance with ethical standards}

Conflict of interest JW Coliins has received advisory role fees from Medtronic and $\mathrm{J} \& \mathrm{~J}$, speakers fees from Intuitive and research grants from Intuitive Surgical and Medtronic in the last 5 years. P Wisc has no potential conflicts of interest to report. 
Ethical approval The article is a review article and no research involving human participants and/or animals was completed.

Informed consent No informed consent was required from patients as this was a review article.

Open Access This article is distributed under the terms of the Creative Commons Attribution 4.0 International License (http://creativeco mmons.org/licenses/by/4.0/), which permits unrestricted use, distribution, and reproduction in any medium, provided you give appropriate credit to the original author(s) and the source, provide a link to the Creative Commons license, and indicate if changes were made.

\section{References}

1. Fried MP, Gallagher AG, Satava RM (2004) Training to proficiency: aircraft to OR. Arch Otolaryngol Head Neck Surg 130(10):1145-1146

2. Nance JJ (2008) Why Hospitals should fly - the ultimate flight plan to patient safety and quality care. Second River Healthcare Press, Bozeman

3. Collins JW, Levy J, Stefanidis D, Gallagher A, Coleman M, Cecil T et al (2019) Utilising the Delphi process to develop a proficiency-based progression train-the-trainer course for robotic surgery training. Eur Urol 75(5):775-785

4. Ahmed K, Khan R, Mottrie A, Lovegrove C, Abaza R, Ahlawat $\mathrm{R}$ et al (2015) Development of a standardised training curriculum for robotic surgery: a consensus statement from an international multidisciplinary group of experts. BJU Int 116(1):93-101

5. ECRI Institute (2014) Top 10 Health Technology Hazards for 2015. Health Devices. https://www.ecri.org/Resources/White papers_and_reports/Top_Ten_Technology_Hazards_2015.pdf. Accessed Oct 2019

6. Fisher RA, Dasgupta P, Mottrie A, Volpe A, Khan MS, Challacombe B et al (2015) An over-view of robot assisted surgery curricula and the status of their validation. Int J Surg 13:115-123

7. Dyer C (2001) Bristol inquiry: Bristol inquiry condemns hospital's "club culture". BMJ 323(7306): 181

8. Aboumatar HJ, Weaver SJ, Rees D, Rosen MA, Sawyer MD, Pronovost PJ (2017) Towards high-reliability organising in healthcare: a strategy for building organisational capacity. BMJ Qual Saf 26(8):663-670

9. Reason J (2000) Human error: models and management. BMJ 320(7237):768-770

10. Raison N, Wood T, Brunckhorst O, Abe T, Ross T, Challacombe B et al (2017) Development and validation of a tool for non-technical skills evaluation in robotic surgery-the ICARS system. Surg Endosc 31(12):5403-5410

11. Collins JW, Dell'Oglio P, Hung AJ, Brook NR (2018) The importance of technical and non-technical skills in robotic surgery training [Figure presented]. Eur Urol Focus 4(5):674-676

12. Lancet The (2008) WHO's patient-safety checklist for surgery. Lancet 372(9632):1. https://doi.org/10.1016/S0140 $-6736(08) 60964-2$

13. Volpe A, Ahmed K, Dasgupta P, Ficarra V, Novara G, Van Der Poel H et al (2015) Pilot validation study of the European association of urology robotic training curriculum. Eur Urol. 68(2):292-299

14. Mottrie A, Novara G, van der Poel H, Dasgupta P, Montorsi F, Gandaglia G (2016) The European association of urology robotic training curriculum: an update. Eur Urol Focus 2(1):105-108

15. Gaba DM (2004) The future vision of simulation in health care. Qual Saf Health Care. 13(Suppl 1):i2-i10
16. Ziv A, Root Wolpe P, Small SD, Glick S (2003) Simulationbased medical education: an ethical imperative. Acad Med 78(8):783-788

17. Haynes AB, Weiser TG, Berry WR, Lipsitz SR, Breizat A-HS, Dellinger EP et al (2009) A surgical safety checklist to reduce morbidity and mortality in a global population. $\mathrm{N}$ Engl $\mathrm{J}$ Med. 360(5):491-499

18. Veronesi G, Dorn P, Dunning J, Cardillo G, Schmid RA, Collins $\mathrm{J}$ et al (2018) Outcomes from the Delphi process of the thoracic robotic curriculum development committee. Eur J Cardiothorac Surg 53(6):1173-1179. https://doi.org/10.1093/ejcts/ezx466

19. Lovegrove C, Novara G, Mottrie A, Guru KA, Brown M, Challacombe B et al (2016) Structured and modular training pathway for robot-assisted radical prostatectomy (RARP): validation of the RARP assessment score and learning curve assessment. Eur Urol 69(3):526-535

20. Sinclair P, Kable A, Levett-Jones T (2015) The effectiveness of internet-based e-learning on clinician behavior and patient outcomes: a systematic review protocol. JBI Database Syst Rev Implement Rep 13(1):52-64. https://doi.org/10.11124/jbisr ir-2015-1919

21. Sotelo RJ, Haese A, Machuca V, Medina L, Nuñez L, Santinelli F et al (2016) Safer surgery by learning from complications: a focus on robotic prostate surgery. Eur Urol 69(2):334-344

22. Raison N, Ahmed K, Abe T, Brunckhorst O, Novara G, Buffi N et al (2018) Cognitive training for technical and non-technical skills in robotic surgery: a randomised controlled trial. BJU Int 122(6):1075-1081

23. Badash I, Burtt K, Solorzano CA, Carey JN (2016) Innovations in surgery simulation: a review of past, current and future techniques. Ann Transl Med 4(23):453

24. MacCraith E, Forde JC, Davis NF (2019) Robotic simulation training for urological trainees: a comprehensive review on cost, merits and challenges. J Robot Surg 13(3):371-377

25. Sotelo RJ, Astigueta JC, Carmona OJ, De Andrade RJ, Moreira OE (2009) Chicken gizzard: a new training model for laparoscopic urethrovesical anastomosis. Actas Urol Esp 33(10):1083-1087

26. Ghazi A, Campbell T, Melnyk R, Feng C, Andrusco A, Stone J et al (2017) Validation of a full-immersion simulation platform for percutaneous nephrolithotomy using three-dimensional printing technology. J Endourol 31(12):1314-1320

27. Vávra P, Roman J, Zonča P, Ihnát P, Němec M, Kumar J et al (2017) Recent development of augmented reality in surgery: a review. J Healthc Eng 2017:4574172. https://doi. org/10.1155/2017/4574172

28. Anvari M (2007) Remote telepresence surgery: the Canadian experience. Surg Endosc 21:537-541

29. Anvari M, McKinley C, Stein H (2005) Establishment of the world's first telerobotic remote surgical service: for provision of advanced laparoscopic surgery in a rural community. Ann Surg 241:460-464

30. Anvari M (2005) Reaching the rural world through robotic surgical programs. Eur Surg 37:284-292

31. Sebajang H, Trudeau P, Dougall A et al (2006) The role of telementoring and telerobotic assistance in the provision of laparoscopic colorectal surgery in rural areas. Surg Endosc 20(9):1389-1393

32. Jung JJ, Jüni P, Lebovic G, Grantcharov T (2018) First-year analysis of the operating room black box study. Ann Surg. https://doi. org/10.1097/SLA.0000000000002863

33. Hung AJ, Chen J, Jarc A, Hatcher D, Djaladat H, Gill IS (2018) Development and validation of objective performance metrics for robot-assisted radical prostatectomy: a pilot study. J Urol 199(1):296-304 
34. MacCraith E, Forde JC, Davis N (2019) Robotic simulation training for urological trainees: a comprehensive review on cost, merits and challenges. J Robot Surg 13(3):371-377

35. Gómez Ruiz M, Alfieri S, Becker T et al (2019) Expert consensus on a Train-the-Trainer curriculum for robotic colorectal surgery. Color Dis 21(8):903-908
36. Collins J, Akre O, Challacombe B, Karim O, Wiklund P (2015) Robotic networks: delivering empowerment through integration. BJU Int 116(2):167-168

Publisher's Note Springer Nature remains neutral with regard to jurisdictional claims in published maps and institutional affiliations. 